\title{
Synchrophasor Communication over Internet: Performance Analysis of different Methods based on Real Experiences
}

\author{
Pablo Leibovich, Student Member, IEEE, Fernando Issouribehere, Member, IEEE, and Juan Barbero \\ IITREE-LAT Universidad Nacional de La Plata \\ La Plata, Argentina \\ pabloleibo@iitree-unlp.org.ar
}

\begin{abstract}
This paper presents a theoretical description and a practical experience in different synchrophasor communication methods applied in real implementations of PMUs connected over both a wired and a mobile internet link. The analyzed methods are proposed in the IEEE Standard C37.118.2-2011 and are the most used in the literature and real applications including commercial PMUs. A complete description is presented and the main characteristics, advantages and disadvantages, including network efficiency algorithms that can affect PMU link are discussed. As PMUs are being applied on existing power systems in developing countries, where dedicated Ethernet or fiber-optic links are not always available, wired internet connections and GSM/GPRS usually become the only possible solutions. Performance of PMUs over Internet, in terms of data frames quality and latency, is analyzed by means of tests designed for this purpose, and statistical analysis of results from real experiences is presented and compared with real applications requirements.
\end{abstract}

Index Terms - Internet, latency, phasor measurement unit (PMU), synchrophasor, transmission methods.

\section{INTRODUCTION}

Interconnected electrical power systems are one of the most important infrastructures of any country or region. Because of its critical importance, controlling its state and being able to estimate the system behavior are two of the main objectives of any system administrator [1].

Typically, to measure and analyze the system, Supervisory Control and Data Acquisition (SCADA) was used. However, measurements obtained with SCADA have important limitations because of a limited temporal resolution, high latency and lack of time synchronization.

In order to measure the stationary state, and to implement automatic power control system, Phasor Measurement Units (PMUs) are used. A PMU is a device that provides synchronized measurements of real-time phasors related to the location where it is installed. Synchronization is obtained by applying the universal coordinated time (UTC) and timing signals obtained from the Global Positioning System (GPS).
This synchronization allows the control system to obtain phasors from different points of the network having common phase relationship between them. This is why they are called Synchrophasors.

The typical structure of these monitoring systems involves not only PMUs but also Phasor Data Concentrators (PDCs). PDCs receive data from different PMUs, aligning and processing the data so that, finally, measurements result available for user process and analysis.

In order to characterize temporal resolutions and latency, IEEE Standard C37.118.1-2011 [2] and its amendments define the maximum acceptable data output reporting latency for PMUs. To define latency requirements for the PDCs, IEEE Standard C37.244-2013 [3] specifies different latencies referred to the PDC data input to data output delays. To accomplish these requirements, a good communication infrastructure is needed and any failure or uncontrolled delay in the communication link will affect the monitoring and control process.

The IEEE Standard C37.118.2 - 2011 [4] defines different messages, including the format and contents of them, for communication between PMUs and PDCs. However, it only describes different communication methods that can be used for the network without specifying a unique link to be used. In the state-of-art of PMUs and PDCs, serial and Ethernet links are the most used for this application, being the second the most used in Wide Area Monitoring Systems (WAMS), Wide Area Monitoring and Control Systems (WAMC) and Wide Area Monitoring, Protection and Control (WAMPAC). In this case, TCP (Transmission Control Protocol) and UDP (User Datagram Protocol) are the common protocols applied for data transfer.

As PMUs networks get wider or installed on older power systems or electrical distribution networks, dedicated Ethernet or fiber-optic links availability is reduced, so that shared networks, like commercial wired internet connections and GSM/GPRS connections become an interesting alternative. This alternative is also the main option in developing countries 
such as Argentina, where power system stations are in remote places with unavailable dedicated data links. In this paper, two synchrophasor communication methods are considered and they were tested in real conditions using wired internet connections and GSM/GPRS. In order to evaluate the typical protocols, TCP connection, based on a client-server topology, was implemented and is called the "commanded method" in the rest of the paper. Also, UDP connection, based on a multicast topology, was implemented and is called the "spontaneous method" in the rest of the paper.

Additionally, this paper shows statistical analysis of the communication performance, showing the advantages and disadvantages of each method and different errors that appear when internet links are used. Delays of data over internet are analyzed and compared to the maximum allowable delays in PMUs based visualization systems and control systems.

The structure of the paper is as follows. Section II provides a brief description of each communication method. Section III presents the platform implemented for both methods and the performed tests. Then, Section IV presents and describes the experimental results. Section V analyzes the application of each communication method on different phasor based systems. Finally, Section VI concludes this paper.

\section{COMMUNICATION METHODS}

The most used communication links for data transference between PMUs and PDCs are based on IP networks. If those links are analyzed with the OSI Model [5], IP addressing method is used for the network layer and TCP or UDP for the Transport Layer.

\section{A. TCP transmission method}

TCP protocol is based on client-server architecture with a connection oriented protocol. This means that the connection requires handshaking to setup and maintain an end-to-end connection. This method establishes a connection to transfer commands from the PDC to the PMU and data in the other way. In this case, PMU works as a server so the client (the PDC) must know the IP address of the server (the PMU) in order to establish the connection and transfer data.

Commercial wired internet connections or GSM/GPRS internet connections are usually based on dynamic IP addressing. This type of IP address management causes the IP addresses of PMUs installed on remote places to be typically unknown. This is the main reason why PMUs publish their address by different methods including Dynamic Domain Name System (DDNS) or periodic IP transference.

As a typical TCP data transfer, PMU data transfer based on TCP has different communication control and security methods, based on detecting lost data packets and rearranging unordered packets. These administration capabilities require, as a consequence, more bandwidth and data rate.

In order to obtain better performance and to reduce the bandwidth usage, TCP protocol has different methods to reduce data transference. One of the common methods is called Nagle's Algorithm [6]. This algorithm states that if few bytes have to be sent (less than a full packet data) and there is already some unacknowledged data in transit, the data sender can wait until either there is more data to be sent (enough to make a full-sized TCP data packet) or the receiver side acknowledges all the outstanding data, so there is no longer any data in transit.

Usually, this is an efficient method to reduce bandwidth usage for typical internet applications. However, in real-time applications where data must be transmitted immediately the Nagle's Algorithm can generate data availability problems. PMUs transmissions over internet can be affected by different network nodes applying this type of techniques.

\section{B. UDP transmission method}

UDP protocol is based on a connectionless communication model. This means that both sides of the link transmit data without handshaking mechanisms. Comparing this method with TCP, there are no available methods for data transference control and security. UDP provides, only, error control data to detect erroneous transmissions. As a consequence, less bandwidth and data rate than the TCP method is required.

PMUs usually implement two types of UDP based communications. The first one is based, as the TCP method, on client-server architecture. PMU works as a server and is controlled by the PDC (the client) with command frames. The second type of UDP based communications is called Spontaneous Data Transmission method in [4].

Spontaneous UDP method is based on a unidirectional data path. In this case, data is transmitted from a PMU to predefined PDCs without commands or other messages in the other way. As a consequence, it must be known, only, the IP addresses of PDCs to be configured in the PMU. This situation is much easier for a monitoring and control system where PMUs are installed in remote places, connected via wired internet or GSM/GPRS connections, and PDCs are installed at places where different internet service providers with fixed IP addressing service are available.

In this case, PMU sends automatically data frames to predefined IP addresses. PDCs cannot send commands or configuration frame requirements to PMUs. For this reason the PMU sends periodically its configuration frame, typically once per minute. This method is available on various commercial PMUs [7]-[9].

Spontaneous method not only reduces the required bandwidth. As there are no transmissions to PMUs, they can be installed behind firewalls or similar systems, blocking the incoming traffic. As a result, the system security is increased obtaining, e.g., immunity to denial of service (DoS) attacks.

In Table I, the PMU data frame size, the minimum packet size, and the required bandwidth for 6 synchrophasors and 50 frames/s for both TCP and UDP protocols are shown. Measurements are supposed to be transmitted in floating point format.

TABLE I. TCP AND UDP DATA SIZE AND BANDWITH.

\begin{tabular}{|c|c|c|}
\hline Description & TCP & UDP \\
\hline PMU Message Frame (payload) & 74 bytes & 74 bytes \\
\hline Minimum header size & 20 bytes & 8 bytes \\
\hline Minimum packet size & 94 bytes & 82 bytes \\
\hline Minimum Bandwidth & $59.6 \mathrm{kbps}$ & $54.4 \mathrm{kbps}$ \\
\hline
\end{tabular}




\section{PMU PLATFORM AND PERFORMED TESTS}

In order to obtain results from a real experience, the PMU communication methods were implemented in both a real PMU hardware and in a PDC running on a personal computer.

The PMU was installed in Concordia, Argentina, $370 \mathrm{~km}$. far from the PDC. The commercial wired internet connection has 12 network nodes between the PMU and the PDC while the GSM/GRPS has 9 network nodes.

Communication links were analyzed by running two different tests designed to show different phenomena taking part on the PMU to PDC data transfer. It is important to mention that the communication between the same PMU and PDC was previously tested over a local area network (LAN), and a perfect transmission was obtained.

\section{A. Analysis of received data packets order}

One of the most important tasks of any PDC is to organize received synchrophasors by their timestamp in order to obtain a time based streaming as the data output. To carry out this task, the PDC must be able to rearrange unsorted data packets so the phasors from different PMUs are time-aligned.

To analyze the order of received packets, the test was based on logging, at PDC side of the communication link, the phasors in the order they arrive. To achieve this goal, a network data logger running as a sniffer of the received data was developed to work in parallel to the PDC. The timestamp of the received synchrophasors was analyzed after the data was logged. This test was performed for both communication methods.

\section{B. Analysis of PMU data frames in communication packets}

Communication methods performance is an important parameter for a WAMS, WAMC or WAMPAC system in order to quantify the entire data latency. These systems are based on an architecture including four main components: PMUs, a PDC, PMUs based application software and the communication network.

PMUs connections to the stations and substations bars of power system are the Data Acquisition layer. Data Management is the next layer, where PMUs data is received and processed by PDCs generating time-data frames. Finally, the third layer is the Application layer, where data visualization software and processing and control software process PMUs data for different analysis.

Between each layer, a communication network must transfer data from one layer to the other. As each data is transferred over this network, it must be compatible in terms of bandwidth and latency in order to obtain the data where it is needed and when it is required.

As described before, quality of data packets transferred across the network must be controlled. The PMU data test is proposed to analyze the characteristics of the received data packets when each communication method is used. The analysis includes studying the effects of algorithms for an efficient use of bandwidth and their influence on data latencies.
With the aim of implementing the test, specific PDC software was developed. Each time a data packet arrives, the software decodes it to detect the number of PMU data frames inside. Once each frame is detected, the software detects if it is complete or incomplete and if there is any data error. This task is done by multiple data frame processors working in parallel, one per received data frame.

Finally, the software generates a report showing the number of data frames received on each data packet, the quality of each frame and the number of detected errors or incomplete frames. In Fig. 1 a block diagram of the processing software is shown.

This test was performed during 10 minutes, several times, applying the commanded method and the spontaneous method, using a commercial wired internet connection and a GSM/GPRS based internet connection.

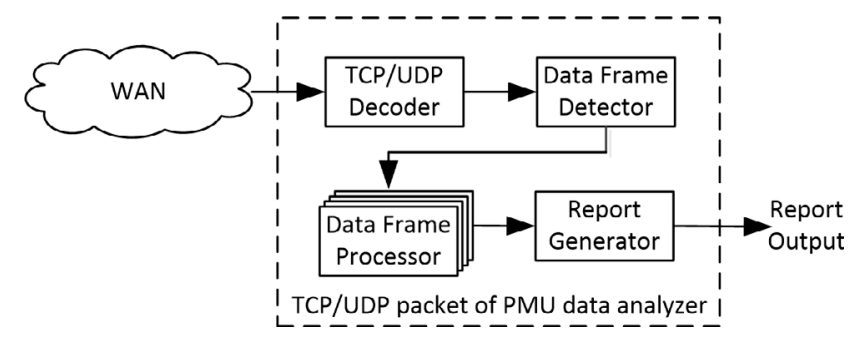

Figure 1. Block diagram of the data packet analyzer software.

\section{EXPERIMENTAL RESULTS}

The order of received packets was analyzed for the commanded and the spontaneous methods, ant it is respectively shown in Fig. 2 and 3. Each case was tested with a configuration of 25 frames/s and 50 frames/s to discard bandwidth limitations.

In these Figures the amplitude of the first received phasor versus the corresponding relative timestamp is shown with the intention of analyzing the chronological order of packets. Relative timestamp means that the first received data frame (not necessarily the first data frame sent by the PMU) corresponds to time zero, and further received timestamps are processed relative to it. As an example, an $x$-axis value of $1 \mathrm{~s}$ corresponds to a timestamp generated $1 \mathrm{~s}$ after the first received timestamp. Negative $x$-axis values mean that the first received timestamp was not the first sent timestamp; that is, at the PDC side, some data frames received are older than the first data frame received.

Fig. 2 shows continuous phasor amplitude without trace crossing, indicating a correct reception of the data, and Fig. 3 shows discontinuous phasor amplitude with trace crossing, indicating the erroneous reception of the data. As a consequence, it can be stated that commanded method did not show any error in the order of received packets while spontaneous method did. This is a consequence of the retransmission and reorder capability of the TCP protocol which is not available in the UDP protocol, so the PDC must reorder them if spontaneous method is applied.

The analysis of PMU data frames on communication packets was performed, as the previous test, for the 


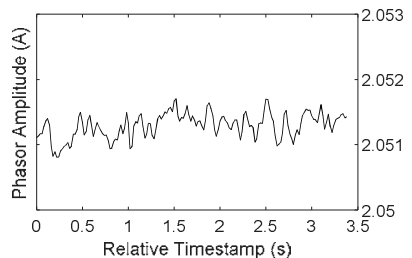

a)

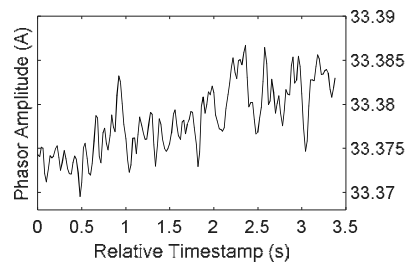

b)
Figure 2. Received packets order - commanded communication method. a) frame rate of 25 frames/s b) frame rate of 50 frames/s.

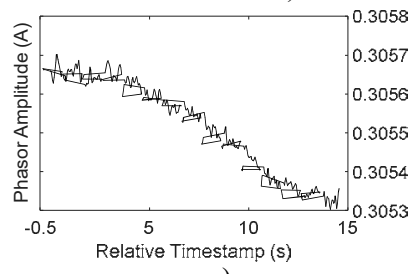

a)

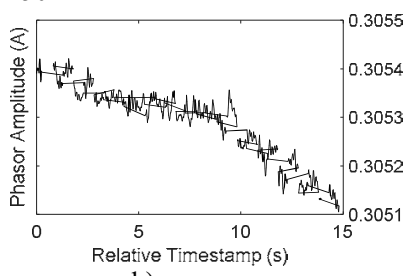

b)
Figure 3. Received packets order - spontaneous communication method. a) frame rate of 25 frames $/ \mathrm{s} \mathrm{b}$ ) frame rate of 50 frames $/ \mathrm{s}$.

commanded and the spontaneous method and for both internet connection types. Each combination of communication method and internet link was tested by logging the received packets over a period of approximately 10 minutes. For each $\log$, the number of data frames received on each packet was registered with the purpose of performing statistical analysis.

Table II shows results obtained from four tests, one for each method and link. Comm. refers to commanded method and Spont. refers to spontaneous method. It can be seen the number of TCP/UDP received packets, the number of received data frames and a classification of packets by the number of data frames received as the payload.

TABLE II. PMU DATA PACKETS AND FRAMES LOG.

\begin{tabular}{|c|c|c|c|c|}
\hline & $\begin{array}{c}\text { Comm. } \\
\text { Wired }\end{array}$ & $\begin{array}{c}\text { Comm. } \\
\text { GSM/GPRS }\end{array}$ & $\begin{array}{c}\text { Spont. } \\
\text { Wired }\end{array}$ & $\begin{array}{c}\text { Spont. } \\
\text { GSM/GPRS }\end{array}$ \\
\hline Total Frames Received & 26406 & 28680 & 26894 & 33263 \\
\hline Packets received & 24971 & 27661 & 26894 & 33263 \\
\hline 1 frames/packet & 24475 & 27226 & 26894 & 33263 \\
\hline 2 frames/packet & 147 & 232 & 0 & 0 \\
\hline 3 frames/packet & 66 & 41 & 0 & 0 \\
\hline 4 frames/packet & 55 & 39 & 0 & 0 \\
\hline 5 frames/packet & 48 & 55 & 0 & 0 \\
\hline 6 frames/packet & 48 & 6 & 0 & 0 \\
\hline 7 frames/packet & 23 & 21 & 0 & 0 \\
\hline 8 frames/packet & 7 & 9 & 0 & 0 \\
\hline 9 frames/packet & 6 & 9 & 0 & 0 \\
\hline 10 frames/packet & 42 & 10 & 0 & 0 \\
\hline Frames with error & 54 & 13 & 0 & 0 \\
\hline
\end{tabular}

Statistical analysis was performed over several tests done for each case. In Fig. 4, the percentage of received packets, on each case, containing a unique data frame is shown.

Table II and Fig. 4 show that data frame merging was not detected in UDP packets generated by spontaneous method, but it was detected in TCP packets of commanded method. As a consequence, it can be concluded that algorithms that improve the use of the bandwidth affect only the commanded method, because it is based on the TCP protocol.
Efficiency algorithms are, in addition, the main reason for which similar results for the same communication method on both a wired connection and a mobile connection are obtained, receiving a mean of $97.5 \%$ of correct packets when TCP is used instead of the $100 \%$ of the UDP protocol.

Based on the statistical results, it can be seen in Fig. 5 the number of packets with 2 to 10 data frames as a percentage of the received packets for the wired connection. It can be concluded that the most important effect of the efficiency algorithms when data frames are merged is to generate packets that for the majority of the cases contain 2 data frames in the same transmission packet.

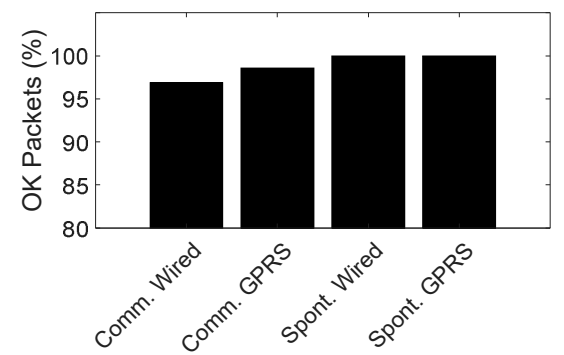

Figure 4. TCP/UDP packets with one Data Frame.

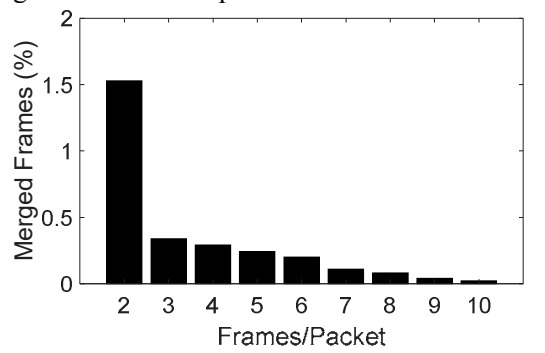

Figure 5. Number of packets with two or more Data Frames.

\section{WAMS, WAMC AND WAMPAC TIME REQUIREMENTS}

As shown in [10]-[12], data latency is critical and has different requirements depending on the application. Results obtained from tests show how, in a TCP based synchrophasor transmission over internet, the effects of different network nodes and algorithms must be considered. Data frame merging in one TCP packet generates unavailability of data over time intervals, which can be analyzed as data latency for the older data frames in the packet. For example, 2 data frames merged on the same packet generates a minimum latency of $20 \mathrm{~ms}$ (on a $50 \mathrm{frames} / \mathrm{s}$ application) which can be worsened by other network and measurement latencies; 5 data frames merged cause a minimum latency of $100 \mathrm{~ms}$.

Dangerous and fast phenomena that can happen in power systems, define strict real-time requirements to WAMC or WAMPAC applications. These real-time requirements determine limits for delays in data transmission, PDC processing, decision making and control actuation.

Delay in data transmission, or latency, is caused by different factors, such as preprocessing data buffering, nodes distance and data processing time. Data latency in a WAMC/WAMPAC system can be estimated as shown in (1)

$$
T_{T}=T_{P}+T_{C}+\frac{P}{R}+\frac{n-1}{S}
$$


where $T_{T}$ is the total delay, $T_{P}$ is the delay associated with signal measurement and processing (30-100 ms), $T_{C}$ is the transmission delay of different communication media (15$150 \mathrm{~ms}), P$ is the amount of data transmitted (6 phasors, floating point format, 384 bits), $R$ is the data rate of the link (100 kbps), $n$ is the number of merged packets (typically 2 ) and $S$ is the synchrophasors data rate (50 frames/s).

Total delay estimation results in about $185 \mathrm{~ms}$ for a dedicated communication link based on a fiber optic network without data merging [13]. As it can be expected, the estimated delay is worsened when internet is considered.

As the worst case, for first swing transient stability, the entire control action must be taken prior to the peak of the forward interarea angle swing (usually 1 to $1.5 \mathrm{~s}$ ) [14]. If the circuit breaker tripping or closing time is considered, synchrophasor based control action must be taken in $0.3 \mathrm{~s}$, including phasor transmission and processing.

These limits can be used as real-time requirements to evaluate each communication method over internet link depending on the synchrophasor application. Commanded method provides always sorted data but with unavailability over time intervals, due to data merging by Nagle's Algorithm. Spontaneous method provides high data availability but unsorted.

For a WAMS system, both methods can be used by applying different algorithms for data processing. For commanded method, older data frames received with the newest one in the same TCP packet can be shown simultaneously or discarded. For spontaneous method, unsorted data frames can be buffered and sorted or, discarded.

For WAMC or WAMPAC systems, data availability is critical to detect dangerous power system conditions and to take control actions. Detection and control algorithms usually require high data availability and have different methods to process unsorted data. Based on these characteristics, maximum accepted latency [12] and suggested communication methods for different applications are described in Table III where $S$ corresponds to suggested method and NS corresponds to non-suggested method.

TABLE III. PMUS ACCEPTED LATENCIES AND COMMUNICATION METHODS OVER INTERNET.

\begin{tabular}{|c|c|c|c|c|c|c|}
\hline \multirow{2}{*}{ Phenomena } & Main application & \multirow{2}{*}{$\begin{array}{c}\text { Latency } \\
\text { (s) }\end{array}$} & \multicolumn{2}{|c|}{ Wired } & \multicolumn{2}{|c|}{ GSM/GPRS } \\
\hline $\begin{array}{c}\text { State } \\
\text { Estimation }\end{array}$ & $\begin{array}{c}\text { Contingency analysis } \\
\text { Power flow }\end{array}$ & 1 & $S$ & $S$ & $S$ & $S$ \\
\hline $\begin{array}{c}\text { Transient } \\
\text { Stability }\end{array}$ & $\begin{array}{c}\text { Load trip } \\
\text { Generation Trip } \\
\text { Islanding }\end{array}$ & 0.3 & $N S$ & $S$ & $N S$ & $S$ \\
\hline $\begin{array}{c}\text { Small } \\
\text { Signal }\end{array}$ & $\begin{array}{c}\text { Oscillation Modes } \\
\text { Modes shape } \\
\text { Damping }\end{array}$ & 1 & $S$ & $S$ & $S$ & $S$ \\
\hline $\begin{array}{c}\text { Dolability } \\
\text { Online PSS update }\end{array}$ & $\begin{array}{c}\text { Capacitor switching } \\
\text { Load shedding } \\
\text { Islanding }\end{array}$ & $1-5$ & $S$ & $S$ & $S$ & $S$ \\
\hline
\end{tabular}

At the output of PDCs, higher data volumes are usually obtained because they generate data packets from multiple PMUs. However, PDCs are typically installed in places where links with higher data rates are available, so the total delay estimation formula and suggestions performed in Table III are still valid.

\section{CONCLUSION}

This paper has presented the two main types of transmission methods from a PMU to a PDC, being them the most common methods implemented on commercial PMUs. A complete description was done showing their main characteristics and differences considering other internet network factors that can affect synchrophasor transmissions.

The performance of each method applied on both a wired and a mobile internet link was studied on the basis of two tests proposed in this paper to analyze different performance factors and data latencies. Data latency was also compared with requirements of typical PMU applications, and the best communication method to be applied, when internet connection is used as the communication link, was proposed.

For PMU transmissions over internet, obtained results prove better performance when spontaneous method is applied. In addition, performed tests confirmed that the effects of the efficiency algorithms applied at each network node, which cannot be controlled by the PMU based system administrator, are very important in terms of data latency through the link. This is a typical situation for PMU transmissions based on TCP over internet.

\section{REFERENCES}

[1] A. G. Phadke and J. S. Thorp, Synchronized Phasor Measurements and Their Applications, New York, Springer, 2008.

[2] IEEE Standard for Synchrophasor Measurements for Power Systems, IEEE Std. C37.118.1, Dec. 2011.

[3] IEEE Guide for Phasor Data Concentrator Requirements for Power System Protection, Control, and Monitoring, IEEE Std. C37.244, May 2013.

[4] IEEE Standard for Synchrophasor Data Transfer for Power Systems, IEEE Std. C37.118.2, Dec. 2011.

[5] Information technology -- Open Systems Interconnection -- Basic Reference Model: The Basic Model. ISO Std. 7498-1. Jun. 1996.

[6] E. Hall, Internet Core Protocols: The Definitive Guide. Sebastopol, O'Reilly, 2000.

[7] General Electric's Grid Solution, "MiCOM P40 Agile P847 Technical Manual", 2015.

[8] Arbiter Systems, "MODEL 1133A Power Sentinel GPS-Synchronized Power Quality Operation Manual", Jun. 2012.

[9] Schweitzer Engineering Laboratories. "The Synchrophasor Report. Synchrophasors and Communications Bandwidth". Apr, 2010.

[10] M. Chenine, I. Al Khatib, J. Ivanovski, V. Maden, L. Nordström. "PMU Traffic Shaping in IP-Based Wide Area Communication" in Proc. IEEE 2010 International Conference on Critical Infrastructure (CRIS).

[11] B.Yang, K. Katsaros, W. Chai, and G.Pavlou, "Cost-Efficient Low Latency Communication Infrastructure for Synchrophasor Applications in Smart Grids", IEEE Systems Journal, vol. 12 No 1 pp. 948-958, Mar. 2018.

[12] P. Kansal and A. Bose, "Bandwidth and Latency requirements for Smart Transmission Grid Applications", IEEE Trans. on Smart Grids, vol. 3 No. 1 pp. 948-958, Sept. 2012.

[13] D. Cai "Wide Area Monitoring, Protection and Control in the future Great Britain Power System" Ph.D. dissertation, Univ. Manchester, Manchester, 2012.

[14] C. Martinez, M. Parashar, J. Dyer, and J. Coroas "Phasor data requirements for Real Time Wide-Area Monitoring, Control and Protection Applications" Consortium for Electric Reliability Technology Solutions, Berkeley, CA, Jan. 2005. 